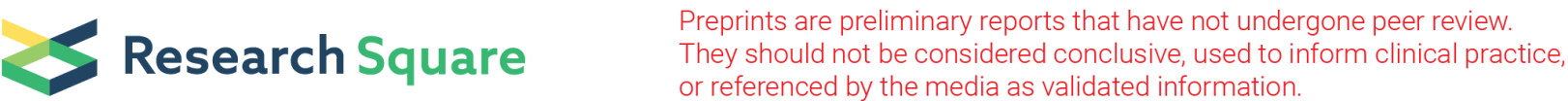

\section{Altered intestinal microbiota are associated with sleep disturbances in patients with minimal hepatic encephalopathy caused by hepatitis B-related liver cirrhosis}

\section{Ming Luo}

People's Hospital of Ningxia Hui Autonomous Region, Ningxia Hui Autonomous Region

Fang-Rui Hu

People's Hospital of Ningxia Hui Autonomous Region, Ningxia Hui Autonomous Region

Yu-Zhen Li

People's Hospital of Ningxia Hui Autonomous Region, Ningxia Hui Autonomous Region

\section{Li Yao}

People's Hospital of Ningxia Hui Autonomous Region, Ningxia Hui Autonomous Region

Sheng-Juan Hu

People's Hospital of Ningxia Hui Autonomous Region, Ningxia Hui Autonomous Region

Fei-Hu Bai ( $\nabla$ baifeihu@hotmail.com )

People's Hospital of Ningxia Hui Autonomous Region, Ningxia Hui Autonomous Region

\section{Research Article}

Keywords: intestinal microbiota, sleep disturbance, minimal hepatic encephalopathy, liver cirrhosis

Posted Date: December 22nd, 2021

DOI: https://doi.org/10.21203/rs.3.rs-1167256/v1

License: (c) (i) This work is licensed under a Creative Commons Attribution 4.0 International License.

Read Full License 


\section{Abstract \\ Objective}

Minimal hepatic encephalopathy (MHE) caused by liver cirrhosis is quite prevalent, and approximately one-half of MHE patients have experience sleep disturbances. This study systematically evaluated the association between sleep disturbances and altered intestinal microbiota in patients with MHE caused by hepatitis B-related liver cirrhosis.

\section{Methods}

Ninety-eight and $45 \mathrm{MHE}$ patients were respectively included in the exploration and validation cohorts. The Chinese version of the Pittsburgh Sleep Quality Index (PSQI) questionnaire was used to evaluate sleep disturbances. The intestinal microbiota of self-collected fecal samples was analyzed using the amplicon sequencing of bacterial $16 \mathrm{~S}$ ribosomal RNA gene.

\section{Results}

MHE patients with sleep disturbances were characterized by lower bacterial diversities and distinct microbial composition in comparison to those without sleep disturbances. The relative abundances of Salivarius, Veillonella, Klebsiella, and Eubacterium were independent predictors of sleep disturbances in MHE patients. In MHE patients with sleep disturbances, the relative abundances of Salivarius and Veillonella were positively correlated with PSQI scores, respectively. Functional modules involved in lipopolysaccharide biosynthesis, as well as protein digestion and absorption, were increased in the microbiome of MHE patients with sleep disturbances.

\section{Conclusion}

Salivarius and Veillonella may be potential diagnostic biomarkers and therapeutic targets for sleep disturbances in MHE patients.

\section{Introduction}

Minimal hepatic encephalopathy (MHE) is a frequently occurring neurological comorbidity due to liver cirrhosis and/or portosystemic shunting, with a spectrum of mild neurocognitive impairments in working memory, psychomotor speed, and attention span ${ }^{1,2}$. MHE prevalence in cirrhotic patients varies between $30 \%$ and $70 \%$, according to epidemiological surveys based on various diagnostic methods in different populations ${ }^{3,4}$. MHE affects daily functioning, impairs driving ability, and is associated with an increased risk of developing overt hepatic encephalopathy ${ }^{5,6}$. 
Sleep disturbances is prevalent, severely influences quality of life, and is related to poor prognosis in MHE patients $^{7,8}$. Existing studies have suggested that altered intestinal microbiota is closely correlated with sleep disturbances in neuropsychiatric diseases such as Parkinson's disease and bipolar disorder ${ }^{9,10}$. Through impairment of the gut-liver-brain axis in cirrhotic patients, altered intestinal microbiota also play significant roles in the pathophysiological mechanisms of $\mathrm{MHE}^{11}$. Thus, we hypothesized that altered intestinal microbiota might be associated with sleep disturbances in patients with MHE caused by liver cirrhosis.

In the presented study, we systematically and comprehensively analysed the intestinal microbiota in MHE patients with and without sleep disturbances, evaluating the association between sleep disturbances and altered intestinal microbiota in patients with MHE caused by hepatitis B-related liver cirrhosis.

\section{Methods}

\section{Patients}

All included MHE patients were recruited from the Department of Gastroenterology in People's Hospital of Ningxia Hui Autonomous Region between January 2019 and January 2021. Hepatitis B-related liver cirrhosis was the underlying etiology of MHE in all of the patients studied. The diagnosis of liver cirrhosis was according to reviews of medical history, radiographic or ultrasound imaging, liver biopsy, and endoscopic manifestations of portal hypertension. Child-Turcotte-Pugh grade was applied to assess the severity of liver cirrhosis.

MHE was diagnosed using the Chinese version of the psychometric hepatic encephalopathy score (PHES), which includes the number connection test- $A$, number connection test-B, line tracing test, digit symbol test, and serial dotting test, as recommended by the updated guideline from the Chinese Society of Hepatology ${ }^{12,13}$. When the score of PHES subtest exceeded mean \pm 2 standard deviations (SD) of the age/education level/gender-matched normal range calculated from 146 healthy Chinese volunteers, the PHES subtest was considered as abnormal ${ }^{13}$. A value of -1 point was assigned to the abnormality in each subtest of PHES, and recruited patients with a total PHES value of $<-4$ points were diagnosed as having $\mathrm{MHE}^{12,13}$.

The following were used as exclusion criteria: clinical manifestations of overt hepatic encephalopathy; recent intake history of probiotics, lactulose, rifaximin in the last 12 weeks; recent intake history of psychotropic drugs in the last 12 weeks; history of recent alcoholism in the last 12 weeks; recent history of overt hepatic encephalopathy, acute gastrointestinal hemorrhage, infection, or electrolyte imbalance in the last 12 weeks; history of primary hepatocellular carcinoma or other malignant tumors; history of fatty liver disease; history of portosystemic shunt or transjugular intrahepatic portosystemic shunt; history of obstructive sleep apnea; obesity (body mass index $[\mathrm{BMl}] \geq 30 \mathrm{~kg} / \mathrm{m}^{2}$ ); neurocognitive diseases including Alzheimer's disease, Parkinson's disease, or ischemic cerebral infarction; vital organ dysfunction including cardiac insufficiency, renal failure, or chronic obstructive pulmonary disease. 
In total, 180 patients with MHE caused by hepatitis B-related liver cirrhosis were recruited in this study, and 120 of whom were randomly assigned to the exploration cohort and the remaining 60 patients were included in the validation cohort (Figure 1). The protocol for this study was approved by the Ethics Committee of People's Hospital of Ningxia Hui Autonomous Region, and it adhered to the ethical principles from the Declaration of Helsinki (6th version, 2008). Before being included in this study, all individuals submitted their written informed consent to participate in it.

\section{Assessment of sleep disturbances}

The Chinese version of the Pittsburgh Sleep Quality Index (PSQI) questionnaire was used to assess sleep disturbances among the included MHE patients. The PSQI is divided into seven subcomponents: sleep quality, sleep latency, sleep duration, sleep efficiency, sleep disturbance, use of sleep medication, and daytime dysfunction ${ }^{14}$. Each subcomponent of the PSQI is scored on a scale of 0 to 3 , with the sum of all subcomponent values resulting in a total score ranging from 0 to 21 . In the Chinese population, a PSQI score of 5 has been demonstrated to be an effective threshold for identifying MHE patients with and without sleep disturbances ${ }^{14}$.

\section{Laboratory tests}

Venous blood samples were taken from all enrolled MHE patients after an overnight fast, and were transmitted for routine hematological, biochemical, and virological testings. Within 20 minutes, plasma ammonia levels were measured by the dry chemistry method with the Vitros 350 Dry Chemistry System (Ortho Clinical Diagnostics, NJ, USA; normal range, 5-35 mol/L). Serum concentrations of HBV-DNA were determined using the Cobas TaqMan HBV-DNA qualitative test (Roche Diagnostics GmbH, Mannheim, Germany), and the lower limit of detection was $1 \mathrm{E}+02$ copies $/ \mathrm{ml}$.

\section{Fecal samples collection and DNA extraction}

Fecal samples were self-collected from all enrolled MHE patients in the hospital using the sterile stool collector (Sarstedt, Numbrecht, Germany) and preserved at $-80^{\circ} \mathrm{C}$ in an ultra-low temperature refrigerator until further processing. The microbial DNA of fecal samples was extracted from $200 \mathrm{mg}$ feces using the QIAamp Fast DNA Stool Mini Kit (Qiagen, Germantown, MD, USA). The concentrations of bacterial DNA were determined by the Nanodrop 2000 spectrophotometer (Thermo Fisher Scientific, Waltham, MA, USA).

\section{Sequencing of $16 S$ ribosomal RNA gene}

The V3-V4 region of the bacterial 16S ribosomal RNA (rRNA) gene was amplified by polymerase chain reaction using the following primer pairs: 341 Forward, CCTACGGGNGGCWGCAG; and 806 Reverse, GGACTACHVGGGTATCTAAT. Amplicons were purified using the QIAquick Gel Extraction Kit (Qiagen, Germantown, MD, USA) and then quantified using the Quant- $-T^{T^{\mathrm{TM}}}$ PicoGreen ${ }^{\mathrm{TM}}$ dsDNA Assay Kit (Thermo Fisher Scientific, Waltham, MA, USA). The MiSeq platform (Illumina, San Diego, CA, USA) was applied to sequence the V3-V4 region of bacterial 16S rRNA genes based on the purified amplicons pool.

\section{Bioinformatic analysis}


The data concerning the sequencing of 16S rRNA gene were analyzed using the Quantitative Insights Into Microbial Ecology software (QIIME2 Version 2020.8) ${ }^{15}$. The sequencing reads were demultiplexed and filtered using the DADA2 software from QIIME2. Using the Vsearch software, the sequencing dataset was clustered to yield operational taxonomic units (OTUs) with criterion of $97 \%$ minimum identity. Chimeric sequences and OTUs with a sequence count of less than $0.005 \%$ of the overall sequence count were identified and removed ${ }^{16}$. An OTU count table with taxonomy was generated based on the detected OTU dataset and accompanying taxonomic assignments. The rarefied OTU table was used to estimate alphadiversity based on species richness. Variations in bacterial diversity across subjects in the different groups were evaluated using beta-diversity analyses based on unweighted UniFrac tests, which were displayed using the principal coordinate analysis (PCOA). The linear discriminant analysis effect size (LEfSe) was applied to determine genera that were significantly different in the relative abundances between two groups. A conditional stepwise logistic regression analysis was performed to identify the independent predictors of sleep disturbances from genera which were found to be significantly different in the LEfSe analysis. Simultaneously, odds ratios (OR) and $95 \%$ confidence intervals $(\mathrm{Cl})$ of the independent predictors were calculated. Furthermore, the analysis of multivariate association with linear models algorithm (MaAsLin) was used to assess the multivariable correlation between clinical covariates and relative abundances of microbial genera ${ }^{17}$. Using the Phylogenetic Investigation of Communities by Reconstruction of Unobserved States (PICRUSt), the metagenomes of the intestinal microbiota were inferred from the sequences of $16 \mathrm{~S}$ rRNA gene ${ }^{18}$. Level 3 of the Kyoto Encyclopedia of Genes and Genomes (KEGG) database pathways was used to generate the predicted functional composition profiles.

\section{Statistical analysis}

Differences in continuous data and bacterial composition between two groups were examined by the Mann-Whitney rank-sum test. The $\chi^{2}$-test or Fisher's exact test were used to compare differences in categorical variables between two groups. The correlation analysis was performed using the partial Spearman's rank-based test. In order to identify the genera that best distinguished MHE patients with and without sleep disturbances, a multivariable stepwise logistic regression analysis was performed using the caret package. A receiver operating characteristic (ROC) curve was established to assess the discriminatory capacity of different genera, and the area under the ROC curve (AUC) was calculated using the $\mathrm{pROC}$ program. Significant differences were defined as those with a two-sided $p<0.05$, and statistical analyses were conducted by the R software (Version 2.15.3).

\section{Results}

\section{Baseline characteristics of included MHE patients}

Based on the inclusion and exclusion criteria, $22 \mathrm{MHE}$ patients were excluded, and $98 \mathrm{MHE}$ patients were ultimately included in the exploration cohort (Figure 1). Fifty-seven percent of included MHE patients in the exploration cohort were male, and the median age was 58 years (range, 29-68 years). The median 
BMI was $22.5 \mathrm{~kg} / \mathrm{m}^{2}$ (range 17-27.5 kg/m²), with a median educational background of 9 years (range, 5-17 years). Of these MHE patients, 82 (84\%), 14 (14\%), and 2 (2\%) were graded as Child-Turcotte-Pugh grade A, B, and C, respectively. Moreover, 70 (71\%) MHE patients had a medical record of previous hospitalization. In total, 89 (91\%) MHE patients have received the treatment of nucleoside analogs including tenofovir and entecavir, and 73 (74\%) of them tested positive for HBV-DNA. Table 1 showed the laboratory values for plasma ammonia, serum sodium, C-reactive protein, hemoglobin, and creatinine.

Meanwhile, 15 MHE patients were excluded, and 45 MHE patients were ultimately included in the validation cohort (Figure 1). The demographic and clinical features of the included MHE patients in the validation cohort were presented in Table 1. Between the exploration and validation cohorts, no statistically significant difference in demographic and clinical features was identified (all $p>0.05$ ) (Table 1).

\section{Table 1}

Demographic and clinical characteristics of included MHE patients in exploration and validation cohorts 


\begin{tabular}{|c|c|c|c|}
\hline Variable & $\begin{array}{l}\text { Exploration } \\
\text { cohort }\end{array}$ & $\begin{array}{l}\text { Validation } \\
\text { cohort }\end{array}$ & $p$-value \\
\hline Number of cirrhotic patients & 98 & 45 & \\
\hline Age(years) & $58(29-68)$ & $56(32-65)$ & 0.093 \\
\hline Gender(male/female) & $56 / 42$ & $26 / 19$ & 0.945 \\
\hline Education(years) & $9(5-17)$ & $9(5-20)$ & 0.147 \\
\hline Body mass index (BMI) & 22.5(17.3-28.7) & 23.4(18.5-29.4) & 0.133 \\
\hline \multicolumn{4}{|l|}{ Aetiology of cirrhosis } \\
\hline Viral hepatitis B & $100 \%$ & $100 \%$ & \\
\hline CTP grade $(\mathrm{A} / \mathrm{B} / \mathrm{C})$ & $82 / 14 / 2$ & $38 / 6 / 1$ & 0.982 \\
\hline Previous hospitalization & 70 & 31 & 0.761 \\
\hline HBV treatment & 89 & 39 & 0.458 \\
\hline entecavir & 74 & 33 & \\
\hline tenofovir & 15 & 6 & \\
\hline HBV-DNA (positive/negative) & $73 / 25$ & $32 / 13$ & 0.675 \\
\hline Ammonia $(\mu \mathrm{mol} / \mathrm{L})$ & $58.6(25.5-76.8)$ & $53.8(22.6-72.5)$ & 0.174 \\
\hline Haemoglobin(g/L) & $115(105-148)$ & $120(112-150)$ & 0.092 \\
\hline Serum sodium(mmol/L) & $136(132-145)$ & $138(133-144)$ & 0.173 \\
\hline C-reactive protein(mg/L) & $6.7(3.5-10.2)$ & $6.2(3.2-9.5)$ & 0.146 \\
\hline Creatinine $(\mu \mathrm{mol} / \mathrm{L})$ & $54(35-70)$ & $49(34-65)$ & 0.182 \\
\hline
\end{tabular}

\section{Sleep quality}

Using the PSQI > 5 as the threshold value, 43 of the 98 MHE patients in the exploration cohort were diagnosed with sleep disturbances, whereas the other $55 \mathrm{MHE}$ patients were identified without sleep disturbances. Simultaneously, 20 MHE patients in the validation cohort were diagnosed with sleep disturbances, and the remaining $25 \mathrm{MHE}$ patients were identified without sleep disturbances (Figure 1). Between the exploration and validation cohorts, there was no significant difference in the proportion of MHE patients with sleep disturbances $(p=0.945)$. Furthermore, no significant distinction concerning the PSQI scores was identified between MHE patients with sleep disturbances in the exploration cohort and those in the validation cohort ( $p=0.434)$. Moreover, the PSQI scores of MHE patients without sleep 
disturbances in the exploration cohort were not significantly different from those in the validation cohort ( $p=0.835)$ (Supplementary Table S1).

\section{Altered microbial composition in MHE patients with sleep disturbances}

The richness of the intestinal microbiota, as evaluated by the count of detected OTUs, was lower in the 43 MHE patients with sleep disturbances than in the $55 \mathrm{MHE}$ patients without sleep disturbances $(p<0.001)$ (Figure 2A). After controlling for age, gender, and BMI, the lower species richness was confirmed. In MHE patients with sleep disturbances, the Shannon indices, which evaluated the richness and evenness of the intestinal microbiota, were significantly lower than in those without sleep disturbances $(p<0.001)$ (Figure 2B). The unweighted UniFrac-based PCoA revealed that MHE patients with sleep disturbances had different microbial compositions, compared to those without sleep disturbances (pseudo-F: 2.65; $p=0.021$ ) (Figure 2C).

At the genus level of intestinal microbiota, 19 bacterial genera were identified that had significantly different abundances in MHE patients with sleep disturbances compared to those without sleep disturbances. The MaAsLin analysis was used to adjust for possible influence factors including age, gender, and BMl; 14 of the 19 genera were confirmed to exhibit differential abundances between MHE patients with and without sleep disturbances (all $p<0.05$ ). Of the remaining genera, decreased abundances of bacteria, including Sutterella, Faecalibacterium, Ruminococcus, Paracoccus, Roseburia, and Actinobacteria, and increased abundances of Lactobacillus, Salivarius, Haemophilus, Clostridium, Klebsiella, Veillonella, Paraprevotella, and Eubacterium, were observed in MHE patients with sleep disturbances (Figure 3A). Notably, in MHE patients with sleep disturbances, Salivarius and Veillonella presented a more than three-fold increase in comparison to those without sleep disturbances $(p<0.001$ and $p=0.004$, respectively) (Figure 3B).

\section{Salivarius distinguished MHE patients with sleep disturbances}

In the exploration cohort, the logistic regression analysis presented that the relative abundances of Salivarius, Veillonella, Klebsiella, and Eubacterium were independent predictors of sleep disturbances in MHE patients (Table 2). A logistic regression model that was composed of relative abundances of these sleep disturbances-associated genera, including Salivarius, Veillonella, Klebsiella, and Eubacterium, could distinguish MHE patients with sleep disturbances from those without sleep disturbances, with an AUC of 0.81 (95\% Cl: $0.74-0.87)$. Moreover, using only Salivarius as a predictor generated an AUC of 0.75 (95\% Cl: 0.68-0.82), and an AUC of 0.64 (95\% Cl: $0.55-0.71)$ was yielded using only Veillonella as a predictor. The AUC was found to be insignificantly different between the logistic regression model and Salivarius ( $p=0.625)$, while the AUC of both the logistic regression model and Salivarius was significantly higher than that of Veillonella ( $p=0.036$ and $p=0.015$, respectively) (Figure 4A). 
In the validation cohort, the logistic regression model that was composed of relative abundances of sleep disturbances-associated genera had an AUC of 0.73 (95\%Cl: $0.65-0.80)$. Salivarius alone yielded an AUC of 0.73 (95\% Cl: $0.64-0.81$ ), and Veillonella alone generated an AUC of 0.61 (95\% Cl: $0.53-0.69)$. In the AUC, there was no statistically significant difference between the logistic regression model and Salivarius $(p=0.966)$, and the AUC of both the logistic regression model and Salivarius was statistically greater than that of Veillonella ( $p=0.029$ and $p=0.032$, respectively) (Figure 4B).

\section{Table 2}

Multivariate logistic regression analysis of sleep disturbances-associated genera in the exploration cohort

\begin{tabular}{|lllll|}
\hline Variables & $\boldsymbol{\beta}$ & OR & $\mathbf{9 5 \%} \mathrm{Cl}$ & $\boldsymbol{p}$-value \\
\hline Lactobacillus & 0.69 & 2.01 & $0.74-5.43$ & 0.175 \\
\hline Clostridium & -0.31 & 0.73 & $0.25-2.22$ & 0.382 \\
\hline Paraprevotella & 0.91 & 2.48 & $0.95-6.42$ & 0.065 \\
\hline Haemophilus & 0.06 & 1.06 & $0.47-2.39$ & 0.692 \\
\hline Eubacterium & 1.28 & 3.59 & $1.51-8.53$ & 0.043 \\
\hline Klebsiella & 1.45 & 4.25 & $1.72-10.33$ & 0.008 \\
\hline Veillonella & 1.75 & 5.24 & $1.29-25.63$ & $<0.001$ \\
\hline Salivarius & 1.63 & 5.58 & $2.34-10.92$ & $<0.001$ \\
\hline OR: odds ratio; Cl: confidence interval. & \\
\hline
\end{tabular}

\section{Correlation between altered intestinal microbiota and sleep disturbances}

In the exploration cohort, after correcting for possible influence factors including age, gender, and BMI using the MaAsLin analysis, the relative abundances of Salivarius were closely correlated with PSQI scores in MHE patients with sleep disturbances (rho $=0.25, p<0.001)$. Furthermore, the relative abundances of Veillonella were also correlated with PSQI scores in these patients (rho=0.38, $p=0.019$ ). Moreover, a positive correlation was identified between plasma ammonia levels and relative abundances of Salivarius in MHE patients with sleep disturbances (rho $=0.69, p<0.001$ ) (Figure $5 \mathrm{~A}$ ).

In the validation cohort, the PSQI scores were correlated with the relative abundances of Salivarius and Veillonella in MHE patients with sleep disturbances (rho $=0.42, p<0.001$ and rho $=0.31, p=0.007$, respectively). Furthermore, there was also a correlation between plasma ammonia levels and relative abundances of Salivarius in these patients ( $r h o=0.56, p=0.033$ ) (Figure 5B). 


\section{Microbial functions altered in MHE patients with sleep disturbances}

In the exploration cohort, the LEfSe analysis found 30 KEGG categories that had significantly different abundances between MHE patients with and without sleep disturbances (LDA score $>2.0, p<0.05$ ). After adjusting for confounders in the MaAsLin analysis, seven functional modules were shown to be substantially related to sleep disturbances in MHE patients (all $p<0.05$ ) (Figure 6). Notably, functional modules related to lipopolysaccharide biosynthesis, as well as protein digestion and absorption, were increased in the microbiome of MHE patients with sleep disturbances.

\section{Discussion}

Accumulating evidences have suggested that altered intestinal microbiota are associated with comorbidities of liver cirrhosis, including MHE and spontaneous bacterial peritonitis ${ }^{19}$. Using the amplicon sequencing of bacterial 16S rRNA gene, we delineated the composition of the intestinal microbiota in MHE patients with and without sleep disturbances. Our findings revealed that the altered intestinal microbiota of MHE patients with sleep disturbances were characterized by lower bacterial diversities and distinct microbial composition in comparison to those without sleep disturbances. The multivariable logistic regression analysis presented that the relative abundances of Salivarius, Veillonella, Klebsiella, and Eubacterium were independent predictors of sleep disturbances in MHE patients. Based on the microbial profiles, a logistic regression model that was composed of these sleep disturbancesassociated genera was able to distinguish MHE patients with sleep disturbances from those without sleep disturbances. The most interesting finding was that higher relative abundances of Salivarius and Veillonella were respectively associated with sleep disturbances, and both may be novel diagnostic biomarkers and therapeutic targets for sleep disturbances in MHE patients.

Relevant investigations by Bajaj et al. have indicated that cirrhotic patients with MHE had higher relative abundances of Veillonella than those without MHE, indicating that the overgrowth of Veillonella may be correlated with MHE presence ${ }^{20,21}$. In this study, we have found that highly enriched Veillonella was associated with sleep disturbances in MHE patients. Meanwhile, functional modules related to lipopolysaccharide production were increased in the microbiome of MHE patients with sleep disturbances, according to the imputed metagenomic analysis by the PICRUSt. This may be explained by the reason that endotoxin produced by Veillonella may be involved in the pathophysiological mechanisms of sleep disturbances in MHE patients. In cirrhotic patients, the overgrowth of Veillonella is associated with increased production of endotoxin, the lipopolysaccharide in the outer membrane of Gram-negative bacteria ${ }^{22}$. Due to impaired intestinal barrier integrity, endotoxin translocates from the intestine to the circulatory system, influences the permeability of the blood-brain barrier, and consequently induces microglial activation in the cerebrum ${ }^{23,24}$. A study using positron emission tomography has found that increased microglial activation in the substantia nigra is related to impaired dopaminergic function in the putamen, which may contribute to sleep disturbances during the idiopathic rapid-eye 
movement (REM) phase ${ }^{25}$. In relevant clinical studies, Martino et al. found that a majority of MHE patients with sleep disturbances had a reduced REM sleep phase than those who had good night sleep ${ }^{26}$. A previous investigation by Bajaj et al. indicated that rifaximin treatment was correlated with lower endotoxemia and improved cognitive function in MHE patients, as well as a slight decrease in the relative abundances of Veillonella ${ }^{21}$. Whether rifaximin could improve sleep quality by reducing the relative abundances of Veillonella requires further studies.

Our study also revealed that highly enriched Salivarius was correlated with sleep disturbances in MHE patients. It has been suggested that the relative abundances of Salivarius were significantly higher in cirrhotic patients with MHE compared to those without MHE, and that the overgrowth of Salivarius was closely associated with MHE presence in patients with liver cirrhosis ${ }^{27}$. Similarly, our investigation indicated a correlation between plasma ammonia levels and relative abundances of Salivarius in MHE patients. Furthermore, we also found that functional modules associated with protein digestion and absorption were highly enriched in the microbiome of MHE patients with sleep disturbances, which has been attributed mainly to the involvement of Salivarius in the production of ammonia ${ }^{27,28}$. Salivarius has a large number of urea catabolism genes capable of activating urease activity, using urea-derived ammonium as its primary source of nitrogen in the intestine, which may contribute to ammonia accumulation and consequently to hyperammonemia ${ }^{28}$. Hyperammonemia induces astrocyte swelling and subsequent low-grade cerebral edema, and further interferes with the inhibition and activation of associated neural nuclei in the parietal cingulate and bilateral anterior, which play significant roles in the REM sleep regulation ${ }^{29,30}$. In the rat model of MHE, Llansola et al. reported that hyperammonemia led to significantly decreased REM sleep phases and increased sleep fragmentation ${ }^{31}$. Lactulose, which has been recommended for the treatment of MHE, has been reported to result in decreased levels of arterial ammonia and improvement of sleep quality in MHE patients ${ }^{32}$. These evidences suggest that lowering ammonia levels by inhibiting the increased abundances of Salivarius might be a possible alternative for the treatment of sleep disturbances in MHE patients.

This study had a few limitations that should be mentioned. First, although the MaAsLin analysis was used to adjust for age, gender, and BMI, the results of this study may have been influenced by other possible confounders, including environmental factors and dietary habits. Second, the PICRUSt, which was used in the 16S rRNA gene sequencing and KEGG pathway analysis, was not able to accurately reveal the microbial composition and function at the species level, compared with the metagenomic sequencing ${ }^{33}$. Third, only patients with MHE caused by hepatitis B-related liver cirrhosis were included in our investigation; as such, findings of our investigation may apply only to these patients and not to those with MHE caused by other aetiologies of liver cirrhosis. Finally, this was a single-center investigation. The findings of this investigation should be confirmed and generalized in larger-scale, multi-center studies with MHE patients from different regions, in part because the composition and structure of the intestinal microbiota varies depending on where the host originates ${ }^{34}$.

\section{Conclusion}


Our study comprehensively and systematically investigated the intestinal microbiota of MHE patients with and without sleep disturbances. We have found that the increased relative abundances of Salivarius and Veillonella were associated with sleep disturbances in MHE patients. Salivarius and Veillonella may be potential diagnostic biomarkers and therapeutic targets for sleep disturbances in patients with MHE caused by hepatitis B-related liver cirrhosis.

\section{Declarations}

\section{ACKNOWLEDGMENTS}

M.L. and F.-R. H. designed the study, analyzed the data, and drafted the manuscript. Y.-Z. L. and L.Y. analyzed and interpreted the data. S.-J. H. and F.-H. B. revised the manuscript for important intellectual content. All authors approved the final version of the manuscript.

\section{DECLARATION OF FUNDING}

This study was supported by the Natural Science Foundation of Ningxia Province (Grant number: 2020AAC03329).

\section{CONFLICTS OF INTEREST}

The authors have no conflicts of interest to declare.

\section{References}

1. Vilstrup, H. et al. Hepatic encephalopathy in chronic liver disease: 2014 practice guideline by the American Association for the Study of Liver Diseases and the European Association for the Study of the Liver. Hepatology 60, 715-735 (2004).

2. Dellatore, P., Cheung, M., Mahpour, N. Y., Tawadros, A. \& Rustgi, V. K. Clinical manifestations of hepatic encephalopathy. Clin. Liver Dis. 24, 189-196 (2020).

3. Han, W., Zhang, H., Han, Y. \& Duan, Z. Cognition-tracking-based strategies for diagnosis and treatment of minimal hepatic encephalopathy. Metab. Brain Dis. 35, 869-881 (2020).

4. Elsaid, M. I. \& Rustgi, V. K. Epidemiology of hepatic encephalopathy. Clin. Liver Dis. 24, 157-174 (2020).

5. Riggio, O. et al. A model for predicting development of overt hepatic encephalopathy in patients with cirrhosis. Clin. Gastroenterol. Hepatol. 13, 1346-1352 (2005).

6. Bajaj, J. S. et al. Minimal hepatic encephalopathy and mild cognitive impairment worsen quality of life in elderly patients with cirrhosis. Clin. Gastroenterol. Hepatol. 18, 3008-3016 (2020).

7. Labenz, C. et al. Prospective evaluation of the impact of covert hepatic encephalopathy on quality of life and sleep in cirrhotic patients. Aliment. Pharmacol. Ther. 48, 313-321 (2018). 
8. Samanta, J. et al. Correlation between degree and quality of sleep disturbance and the level of neuropsychiatric impairment in patients with liver cirrhosis. Metab. Brain Dis. 28, 249-259 (2013).

9. Heintz-Buschart, A. et al. The nasal and gut microbiome in Parkinson's disease and idiopathic rapid eye movement sleep behavior disorder. Mov. Disord. 33, 88-98 (2018).

10. Evans, S. J. et al. The gut microbiome composition associates with bipolar disorder and illness severity. J. Psychiatr. Res. 87, 23-29 (2017).

11. Bajaj, J. S. The role of microbiota in hepatic encephalopathy. Gut Microbes 5, 397-403 (2014).

12. Xu, X. Y. et al. Chinese guidelines on management of hepatic encephalopathy in cirrhosis. World J. Gastroenterol. 25, 5403-5022 (2019).

13. Li, S. W. et al. Psychometric hepatic encephalopathy score for diagnosis of minimal hepatic encephalopathy in China. World J. Gastroenterol. 19, 8745-8751 (2013).

14. Tsai, P. S. et al. Psychometric evaluation of the Chinese version of the Pittsburgh Sleep Quality Index (CPSQI) in primary insomnia and control subjects. Qual. Life Res. 14, 1943-1952 (2005).

15. Kuczynski, J. et al. Using QIIME to analyze 16S rRNA gene sequences from microbial communities. Curr. Protoc. Microbiol. Chapter 1, Unit 1E.5 (2012).

16. Navas-Molina, J. A. et al. Advancing our understanding of the human microbiome using QIIME. Methods Enzymol. 531, 371-444 (2013).

17. Morgan, X. C. et al. Dysfunction of the intestinal microbiome in inflammatory bowel disease and treatment. Genome Biol. 1, R79 (2012).

18. Langille, M. G. et al. Predictive functional profiling of microbial communities using $16 \mathrm{~S}$ rRNA marker gene sequences. Nat. Biotechnol. 31, 814-821 (2013).

19. Gómez-Hurtado, I., Such, J., Sanz, Y., \& Francés, R. Gut microbiota-related complications in cirrhosis. World J. Gastroenterol. 20, 15624-15631 (2014).

20. Bajaj, J. S. et al. Linkage of gut microbiome with cognition in hepatic encephalopathy. Am. J. Physiol. Gastrointest. Liver Physiol. 302, G168-G175 (2012).

21. Bajaj, J. S. et al. Modulation of the metabiome by rifaximin in patients with cirrhosis and minimal hepatic encephalopathy. PLoS One 8, e60042 (2013).

22. Deng, Y. D. et al. The intestinal microbial community dissimilarity in hepatitis $B$ virus-related liver cirrhosis patients with and without at alcohol consumption. Gut Pathog. 11, 58 (2019).

23. Benjamin, J., Singla, V., Arora, I., Sood, S., \& Joshi, Y. K. Intestinal permeability and complications in liver cirrhosis: A prospective cohort study. Hepatol. Res. 43, 200-207 (2013).

24. Qin, L. et al. Increased systemic and brain cytokine production and neuroinflammation by endotoxin following ethanol treatment. J. Neuroinflammation 5, 10 (2008).

25. Stokholm, M. G. et al. Assessment of neuroinflammation in patients with idiopathic rapid-eyemovement sleep behaviour disorder: a case-control study. Lancet Neurol. 16, 789-796 (2017).

26. Martino, M. E., Fernández-Lorente, J., Romero-Vives, M., Bárcena, R., \& Gaztelu, J. M. Brain oscillatory activity during sleep shows unknown dysfunctions in early encephalopathy. J. Physiol. Biochem. 70, 
$821-835$ (2014).

27. Zhang, Z. et al. Large-scale survey of gut microbiota associated with MHE Via 16 S rRNA-based pyrosequencing. Am. J. Gastroenterol. 108, 1601-1611 (2013).

28. Chen, Y. Y., Weaver, C. A., \& Burne, R. A. Dual functions of Streptococcus salivarius urease. J. Bacteriol. 182, 4667-4669 (2000).

29. Zwingmann, C. Nuclear magnetic resonance studies of energy metabolism and glutamine shunt in hepatic encephalopathy and hyperammonemia. J. Neurosci. Res. 85, 3429-3442 (2007).

30. Maciel, R. et al. Is REM sleep a paradoxical state?: Different neurons are activated in the cingulate cortices and the claustrum during wakefulness and paradoxical sleep hypersomnia. Biochem. Pharmacol. 191, 114514 (2021).

31. Llansola, M. et al. Progressive reduction of sleep time and quality in rats with hepatic encephalopathy caused by portacaval shunts. Neuroscience 201, 199-208 (2012).

32. Singh, J., Sharma, B. C., Puri, V., Sachdeva, S. \& Srivastava, S. Sleep disturbances in patients of liver cirrhosis with minimal hepatic encephalopathy before and after lactulose therapy. Metab. Brain Dis. 32, 595-605 (2017).

33. Jovel, J. et al. Characterization of the gut microbiome using $16 \mathrm{~S}$ or shotgun metagenomics. Front. Microbiol. 7, 459 (2016).

34. He, Y. et al. Regional variation limits applications of healthy gut microbiome reference ranges and disease models. Nat. Med. 24, 1532-1535 (2018).

\section{Abbreviations}




\begin{tabular}{ll} 
MHE & minimal hepatic encephalopathy \\
\hline PHES & psychometric hepatic encephalopathy score \\
\hline BMI & body mass index \\
\hline PSQI & Pittsburgh Sleep Quality Index \\
\hline OTU & operational taxonomic unit \\
PCoA & principal coordinate analysis \\
\hline LEfSe & linear discriminant analysis effect size \\
\hline MaAsLin & multivariate association with linear models algorithm \\
\hline PICRUSt & Phylogenetic Investigation of Communities by Reconstruction of Unobserved States \\
& \\
\hline KEGG & Kyoto Encyclopedia of Genes and Genomes \\
\hline ROC & receiver operating characteristic \\
\hline AUC & area under the receiver operating characteristic curve
\end{tabular}

\section{Figures}




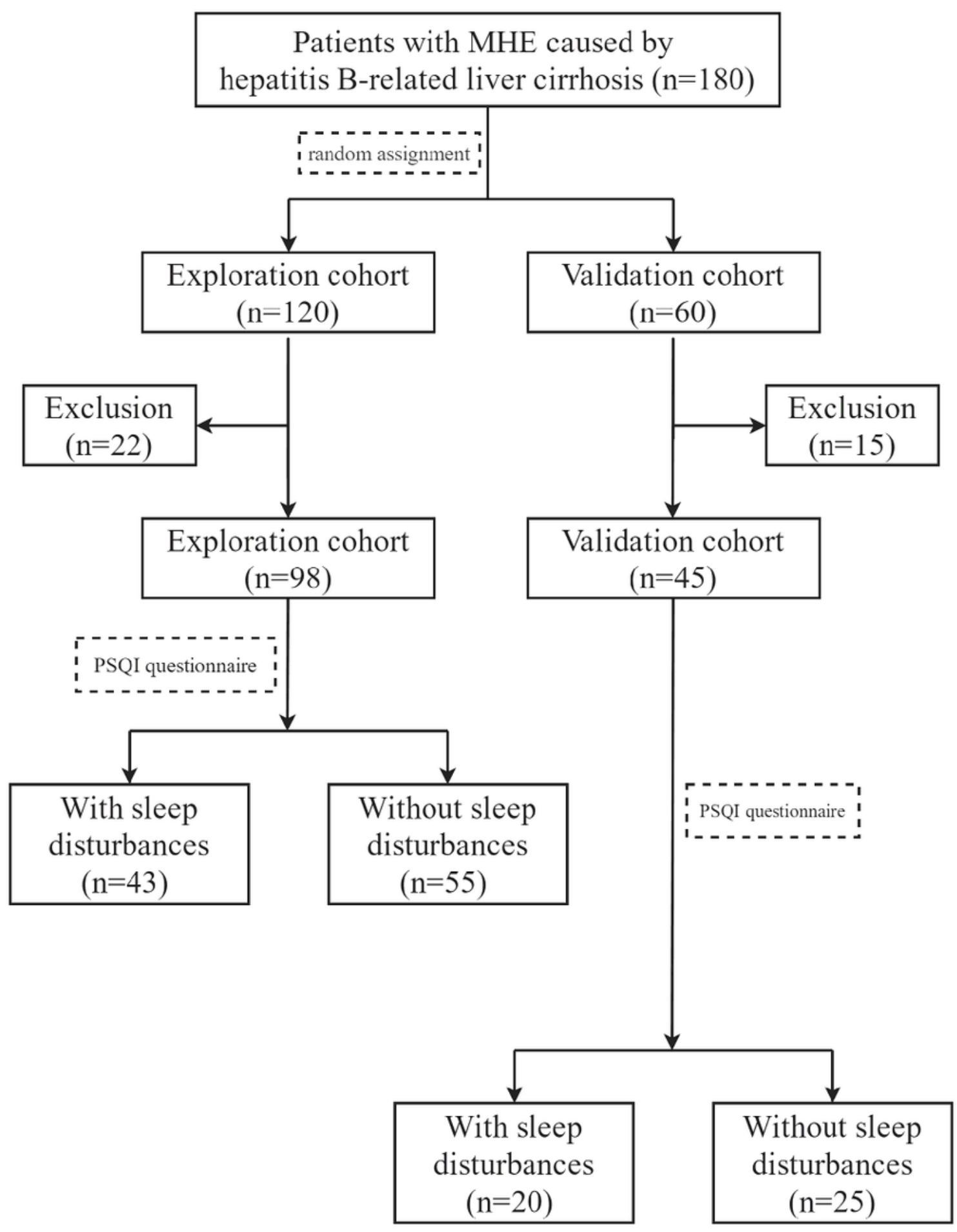

\section{Figure 1}

Flow diagram of minimal hepatic encephalopathy (MHE) patients included in this study and the diagnosis of sleep disturbances using the Pittsburgh Sleep Quality Index (PSQI) questionnaire. 
(A)

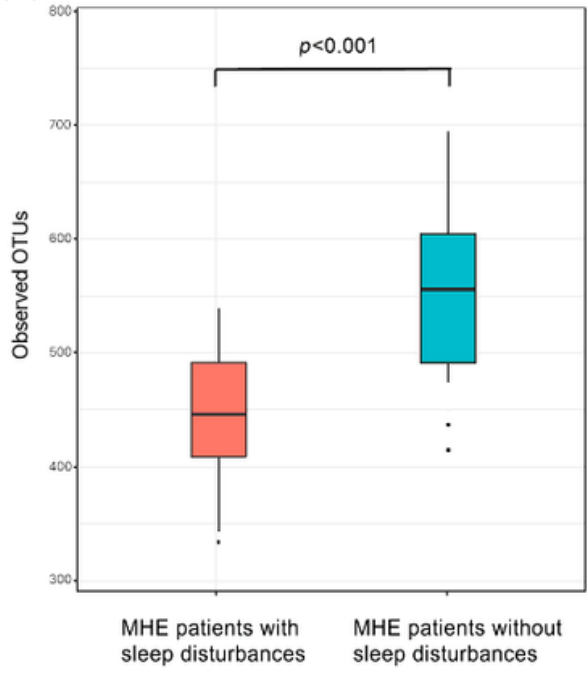

(B)

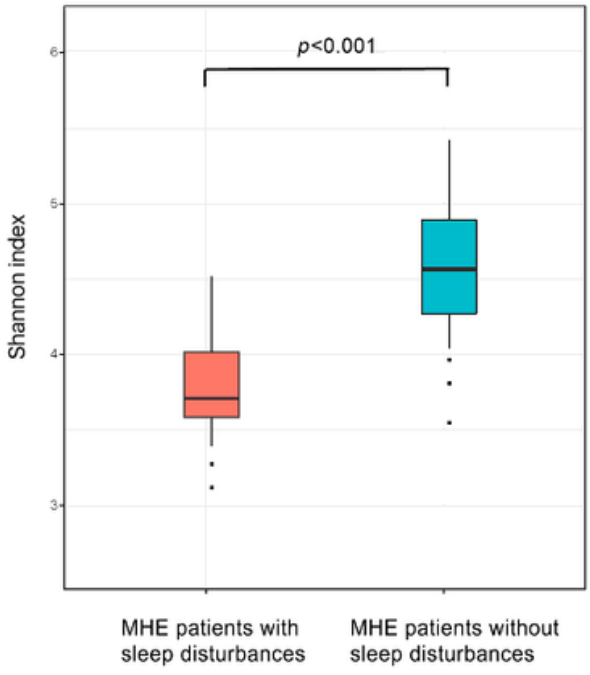

(C)

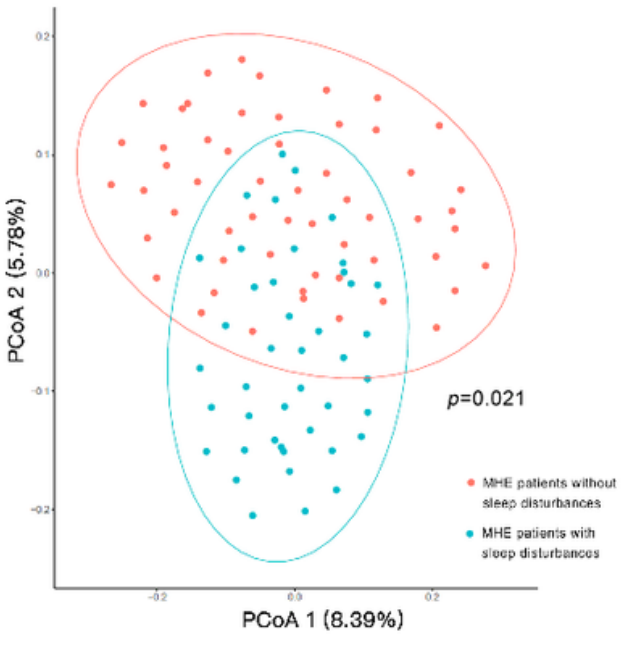

Figure 2

Comparisons of alpha-diversity and beta-diversity between minimal hepatic encephalopathy (MHE) patients with and without sleep disturbances.

(A) The number of observed operational taxonomic units (OTUs) was significantly reduced in MHE patients with sleep disturbances.

(B) Shannon diversity indices were significantly decreased in MHE patients with sleep disturbances.

(C) Principal coordinate analysis (PCoA) based on unweighted UniFrac matrix revealed that the overall composition of intestinal microbiota was different between MHE patients with and without sleep disturbances (pseudo-F: 2.65, $p=0.021$ ). 


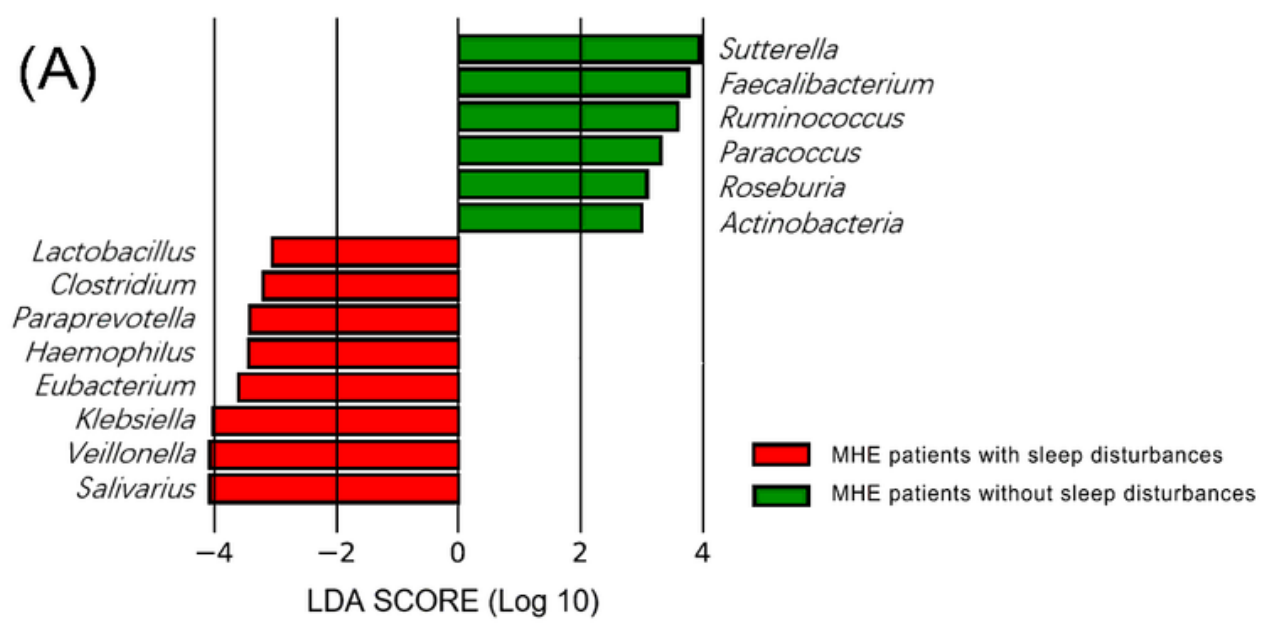

(B)

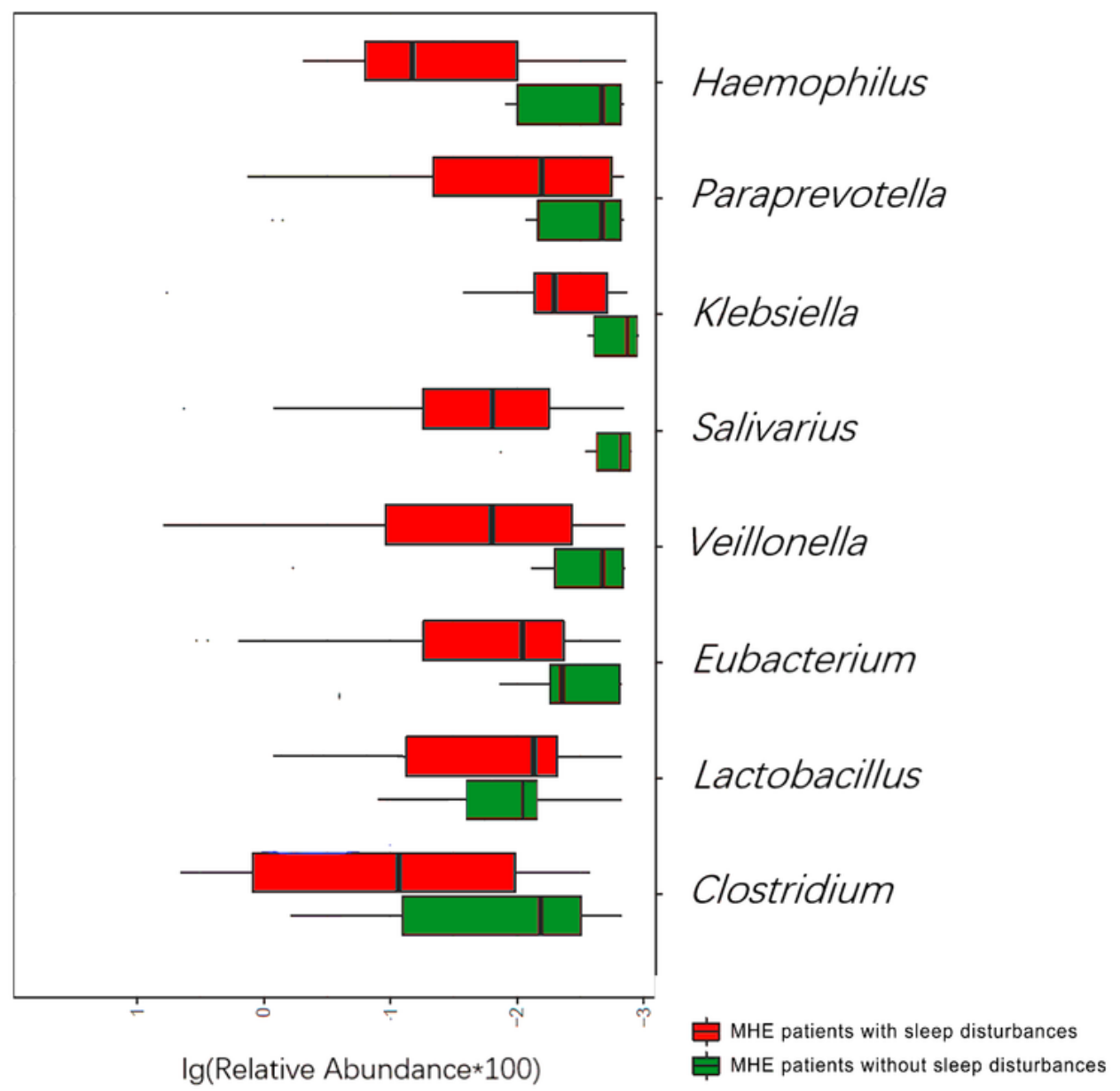

Figure 3

(A) Linear discriminant analysis effect size (LEfSe) revealed that the relative abundances of 14 genera were significantly different between minimal hepatic encephalopathy (MHE) patients with and without sleep disturbances (all $p<0.05$ ). 
(B) Comparison of relative abundances of sleep disturbances-associated genera between MHE patients with and without sleep disturbances (all $p<0.05$ ).
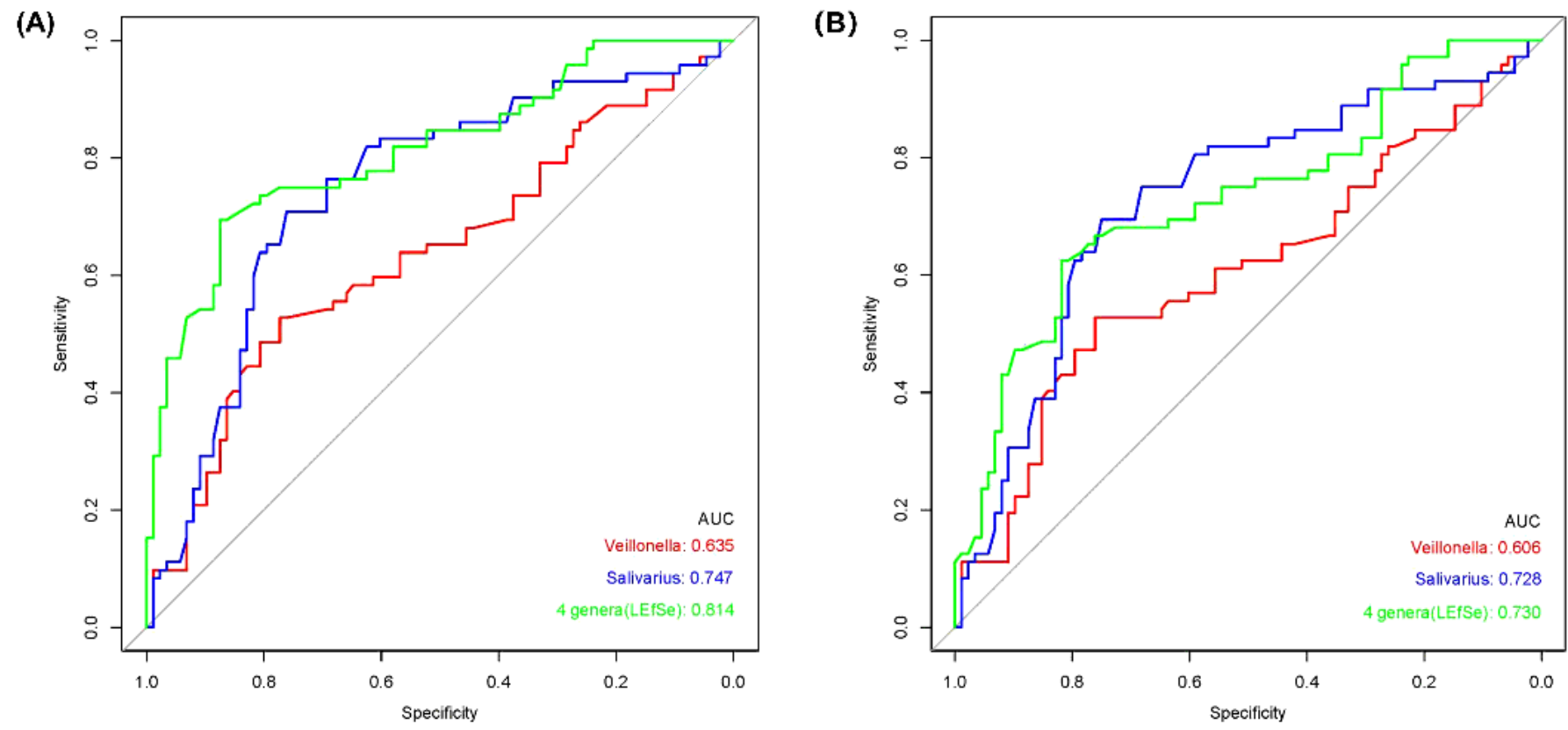

\section{Figure 4}

Multivariable logistic regression model that was composed of relative abundances of four sleep disturbances-associated genera was evaluated by the receiver operating characteristic (ROC) curves, and areas under the ROC curves (AUC) were compared. (A) Data from the exploration cohort. (B) Data from the validation cohort.

(A)

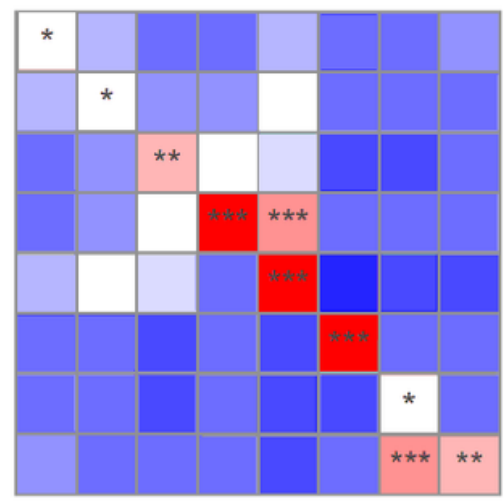

Lactobacillus
Paraprevotella
Clostridium
Salivarius
Veillonella
Klebsiella
Haemophilus
Eubacterium

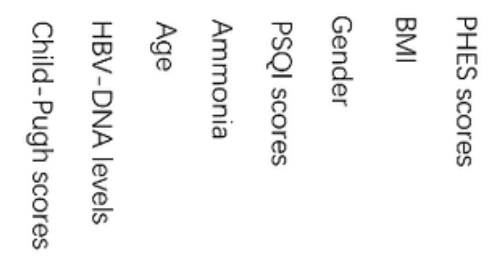

(B)

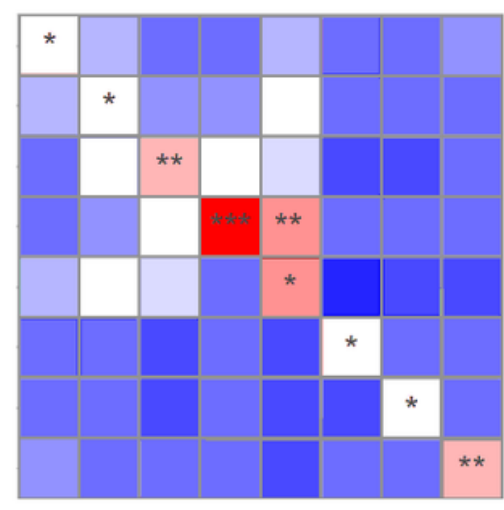

Haemophilus

Clostridium

Paraprevotella

Salivarius

Veillonella

Eubacterium

Lactobacillus

Klebsiella 
Heatmap presented the partial Spearman correlation between sleep disturbances- associated genera and clinical indices in MHE patients with sleep disturbances. (A) Data from the exploration cohort; (B) Data from the validation cohort.

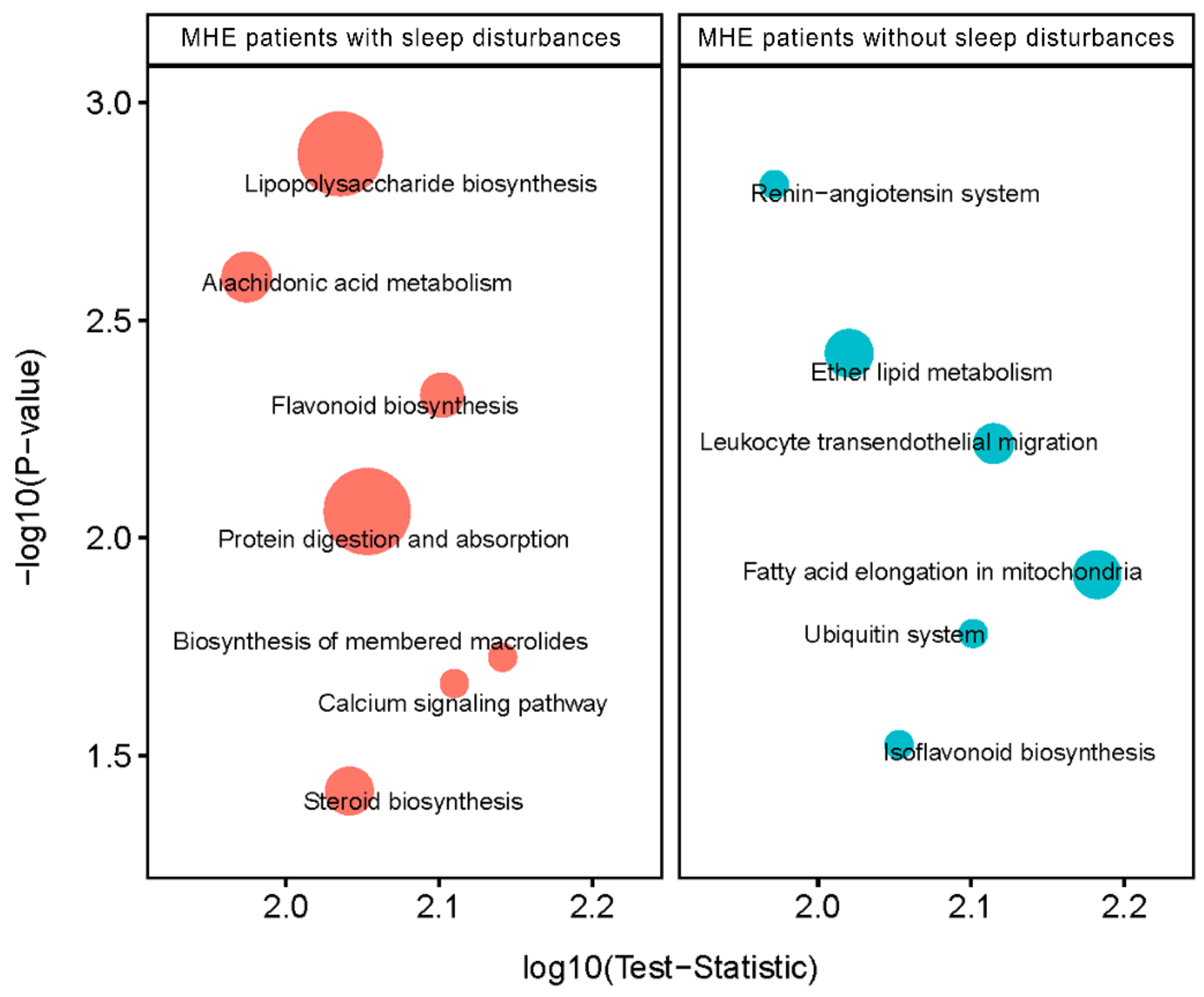

\section{Figure 6}

Functional analysis of predicted metagenomes. Differentially abundant Kyoto Encyclopedia of Genes and Genomes (KEGG) pathways between minimal hepatic encephalopathy (MHE) patients with sleep disturbances and those without sleep disturbances were identified by the linear discriminant analysis effect size (LEfSe) analysis. Circle size corresponds to the relative abundance of each module.

\section{Supplementary Files}

This is a list of supplementary files associated with this preprint. Click to download. 
- SupplementaryTableS1.docx

Page 21/21 